\title{
Clostridium difficile carriage in hospitalized cancer patients: a prospective investigation in eastern China
}

\author{
Wei-Jia Fang ${ }^{1}$, Da-Zhi Jing ${ }^{2}$, Yun Luo ${ }^{2}$, Cai-Yun Fu ${ }^{3}$ Peng Zhao ${ }^{1}$, Jiong Qian', Bing-Ru Tian ${ }^{4}$, Xiao-Gang Chen ${ }^{1}$, \\ Yu-Long Zheng ${ }^{1}$, Yi Zheng ${ }^{1}$, Jing Deng ${ }^{1}$, Wei-Hua Zou ${ }^{5}$, Xue-Ren Feng ${ }^{5}$, Fan-Long Liu ${ }^{1}$, Xiao-Zhou Mou ${ }^{6}$ \\ and Shu-Sen Zheng ${ }^{1 *}$
}

\begin{abstract}
Background: Clostridium difficile carriage has been considered as a potential source for the deadly infection, but its role in cancer patients is still unclear. We aimed to identify the clinical and immunological factors that are related to C. difficile carriage in Chinese cancer patients.

Methods: A total of 400 stool samples were collected from cancer patients who received chemotherapy in three hospitals of eastern China. Bacterial genomic DNA was extracted and two toxin genes ( $t c d A$ and $t c d B$ ) were detected. PCR ribotyping was performed using capillary gel electrophoresis. Concentrations of prostaglandin E2 (PGE2), transforming growth factor beta (TGF- $\beta$ ) and interleukin-10 (IL-10) were measured using enzyme-linked immunosorbent assay (ELISA) kits.

Results: Eighty-two (20.5\%) samples were confirmed to be C. difficile-positive and positive for tpi, $t c d A$, and $t c d B$ genes. The C. difficile-positive rates in patients with diarrhea and no diarrhea were $35 \%$ and $19.7 \%$, respectively $(p=0.09)$. Patients who were younger than 50 years old and were hospitalized for at least 10 days had a C. difficile-positive rate as high as $35 \%$. In contrast, patients who were older than 50 years old and were hospitalized for less than 10 days had a $C$. difficile-positive rate of only 12.7\% ( $p=0.0009)$. No association was found between $C$. difficile carriage and chemotherapy regimen, antibiotic drug use, or immunosuppressive mediators, such as prostaglandin E2 (PGE2), transforming growth factor beta (TGF- $\beta$ ), or interleukin-10 (IL-10). Twelve ribotypes of C. difficile were identified, but none of them belonged to ribotype 027.
\end{abstract}

Conclusions: We conclude that younger patients and those with longer hospitalization stays may be more prone to $C$. difficile carriage. Studies of larger populations are warranted to clarify the exact role of $C$. difficile carriage in hospitalized cancer patients in China.

Keywords: Clostridium difficile, Carriage, Age, Hospitalization days

\section{Background}

Clostridium difficile infection is a hospital-acquired infection, and its prevalence has increased [1]. According to a report by the U.S. Centers for Disease Control and Prevention in 2013, C. difficile infection has been considered as an urgent threat and requires immediate attention. The major risk factors for $C$. difficile infection include the use

\footnotetext{
* Correspondence: shusenzheng@zju.edu.cn

${ }^{1}$ First Affiliated Hospital, School of Medicine, ZheJiang University, 79 Qinchun Road, Hangzhou 310006, China

Full list of author information is available at the end of the article
}

of antibiotics, the use of proton pump inhibitors (PPIs), hospitalization, aging, and conditions that may affect the colonic flora [2-5].

It has been reported that cancer patients have a higher risk for $C$. difficile infection as compared to noncancer patients [6]. However, most information currently available on $C$. difficile infection is from the non-oncologic population. Until now, there is still a lack of comprehensive epidemiological studies of $C$. difficile infection in China, let alone specific investigations on cancer populations. 
Herein, we conducted a comprehensive investigation on $C$. difficile carriage in cancer patients from three hospitals in eastern China. We hoped to identify potential clinical or laboratory factors that are related to C. difficile carriage in a variety of cancer patients.

\section{Methods \\ Patients}

Samples were collected from cancer centers in three hospitals (The First Affiliated Hospital of Zhejiang University, Yu-Yao Hospital, and Hu-Zhou Central Hospital). Each center has about sixty beds and they were expected to admit more than 1,000 cancer patients in total per year. All patients provided written consent, and the study protocol was approved by the Institutional Ethics Committees of all three hospitals, where samples were collected. The first stool sample after admission was collected from each cancer patient. Other information collected from the patients included the following: time of sample collection, age, gender, diagnosis, gastroenterological surgery within the last 3 months, cumulative days of hospitalization within the last 3 months, chemotherapy regimen and antibiotic drug use within the last 3 months, PPI use, diarrhea within the last 3 months, as well as counts of white blood cells and blood albumin. Medical records were reviewed and patient interviews were conducted to assess diarrhea symptoms.

Diarrhea was defined as having three or more loose, watery stool passages during a $24-\mathrm{h}$ period $[7,8]$. Diarrhea was further classified as follows: mild diarrhea, characterized by the absence of signs and symptoms of colitis; moderate diarrhea, characterized by the presence of colitis, fever, and abdominal cramps, usually in the lower quadrants; and severe diarrhea, defined as colitis associated with a leukocyte count greater than or equal to 15,000 cells/l and a serum creatinine level greater than or equal to 1.5 times the premorbid level $[7,8]$.

\section{Isolates from stool specimens and detection of toxin genes}

All stool specimens were treated with alcohol, and the mixture was inoculated into cefoxitin-cycloserine fructose agar (CCFA) plates (Oxoid, Basingstoke) [9]. After incubation for $48 \mathrm{~h}$ at $37^{\circ} \mathrm{C}$ in a GENbag anaerobic chamber (BioMérieux, Marcy l'Etoile, France), the isolates were confirmed to be $C$. difficile based on assays as previously described [10]. Bacterial genomic DNA was extracted using a DNA extraction kit (Qiagen, Inc., Valencia, CA), according to the manufacturer's instructions. The housekeeping gene tpi and the two toxin genes ( $t c d A$ and $B)$ were detected using assays as previously described $[9,11]$. Amplified products were analyzed by electrophoresis with ethidium bromide. DNA sequencing of all polymerase chain reaction (PCR) products was performed according to standard protocols.

\section{PCR ribotyping}

PCR ribotyping was performed using capillary gel electrophoresis with primer pairs as described previously [12]. The size of each peak was determined using Genemapper ID-X software 1.3 (Applied Biosystems). The capillary sequencer-based PCR-ribotyping data were analyzed using the WEBRIBO website (https://webribo.ages.at/) [13].

\section{Detection of immunosuppressive mediators}

Concentrations of prostaglandin E2 (PGE2; MaiBio, Shanghai, China), transforming growth factor beta (TGF- $\beta$; Ebioscience, San Diego, CA, USA), and interleukin-10 (IL-10; Ebioscience, San Diego, CA, USA) were measured using enzyme-linked immunosorbent assay (ELISA) kits. The ELISA kits were purchased from the above companies, respectively, and ELISA was carried out according to the manufactures' instruction.

\section{Statistical analysis}

Statistical analysis was performed by SPSS 20.0 (Chicago, IL, USA). First, an $\mathrm{R} \times \mathrm{C}$ contingency table was established to consolidate the quantitative data. The data were then divided into different groups. The chi-squared test was used to analyze categorical data. The F-test and T-test were conducted for hetero or equal variance analysis. Significance of variables was determined by univariate Cox regression analysis (Figure 1). Statistical significance for all tests was defined as $\mathrm{p}<0.05$.

\section{Results}

A total of 400 samples were collected from April to October, 2013. The numbers of samples obtained from Zhejiang University, Yu-Yao, and Hu-Zhou hospitals were 359, 26, and 15, respectively. There were 259 (65\%) male and 141 (35\%) female patients, and the median age was 57 years old. Further characteristics of the patients and $C$. difficile detection outcomes are listed in Table 1. Among the 82 (20.5\%) C. difficile-positive patients, they were all $t c d A$ and $t c d B$ positive strains $(t c d A+$ and $t c d B+)$ and no toxin gene-deleted strains $(t c d A+$ and $t c d B-$, $t c d A-$ and $t c d B+$ ) were identified. All the 20 diarrheal patients were considered as mild diarrhea. No moderate or severe diarrhea was identified. The $C$. difficile-positive rates in the diarrhea and nondiarrhea patients were $35 \%$ and $19.7 \%$, respectively $(\mathrm{p}=0.09$, as shown in Table 1$)$. No association was found between the $C$. difficile-positive rate and clinical factors, including collection time, sex, diagnosis, PPI use, chemotherapy regimen and gastroenterological surgery within the last 3 months, and antibiotic drug use within the last 3 months. 


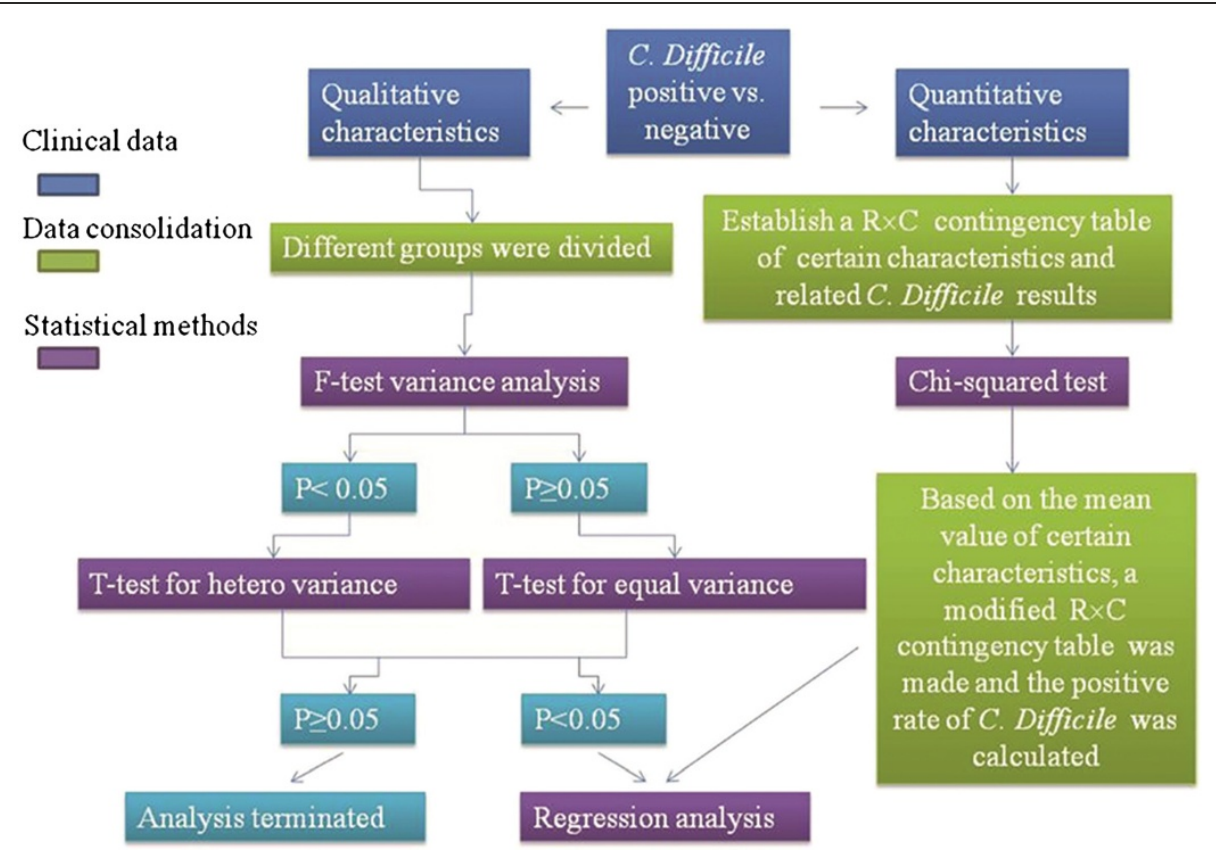

Figure 1 Statistical analysis procedure.

\section{Age and $C$. difficile carriage}

The mean ages of the $C$. difficile-positive and $C$. difficilenegative patients were 54 and 56.7 years old, respectively. The T-test showed that the $C$. difficile infection rate between these two groups was not significantly different $(p=0.06)$. Next, the patients were divided into five consecutive age groups. As shown in Figure 2a, the cancer patients in the two age groups of patients who were younger than 50 years old had a higher infection rate

Table 1 Patient characteristics and C. difficile detection outcomes

\begin{tabular}{lll}
\hline Category & Subdivision & $\begin{array}{l}\text { Number } \\
\text { (percentage, \%) }\end{array}$ \\
\hline Cancer type & Colorectal cancer & $68(17)$ \\
& Noncolorectal digestive & $128(32)$ \\
& tract cancer & \\
& Nondigestive tract cancer & $172(43)$ \\
& Hematological malignancies & $32(8)$ \\
C. difficile Carriage & Positive & $82(20.5)$ \\
& Negative & $318(79.5)$ \\
Diarrhea & Yes & $20(5)$ \\
& C. difficile-positive & $7(35)^{\#}$ \\
& C. difficile-negative & $13(65)$ \\
& No & $380(95)$ \\
& C. difficile-positive & $75(19.7)^{\#}$ \\
& C. difficile-negative & $305(80.3)$ \\
\hline
\end{tabular}

\#The C. difficile-positive rate was greater in diarrhea patients than in non-diarrhea patients; however, the difference was not statistically significant $(p=0.09)$. than the other three groups of patients who were older than 50 years old. Therefore, 50 years old was set as the cut-off value and all of the patients were then grouped into two groups based on this age. The infection rate was $26.9 \%$ in the younger group and $18.2 \%$ in the older group. The difference was statistically significant $\left(x^{2}=4.17\right.$; $\mathrm{p}=0.04)$.

\section{Cumulative days of hospitalization and C. difficile carriage rate}

Next, it was determined if the cumulative number of days in hospital was related to the $C$. difficile positive rate. For the 318 C. difficile-negative patients, the cumulative number of days in hospital (within 3 months of sample collection) was 11.7 days. While for the C. difficile-positive patients, the cumulative number of days in hospital was 19.0 days, which was significantly longer $(t=3.5$, $\mathrm{p}=0.0005)$. Further analysis showed that the difference was more significant when all of the patients were divided into two groups: hospitalization for 10 days or longer and less than 10 days. The $C$. difficile-positive rates were $28.4 \%$ and $13.3 \%$, respectively $\left(x^{2}=13.92 ; \mathrm{p}=0.0002\right)$. So, 10 days of hospitalization was set as the cut-off value for cancer patients.

\section{Combination of age, days of hospitalization, and C. difficile-positive rate}

Using the two cut-off values of 50 years old and 10 days of hospitalization, the 400 cancer patients were divided into four groups and the positive rates of $C$. difficile in the various groups were analyzed. As shown in Figure 2b, 

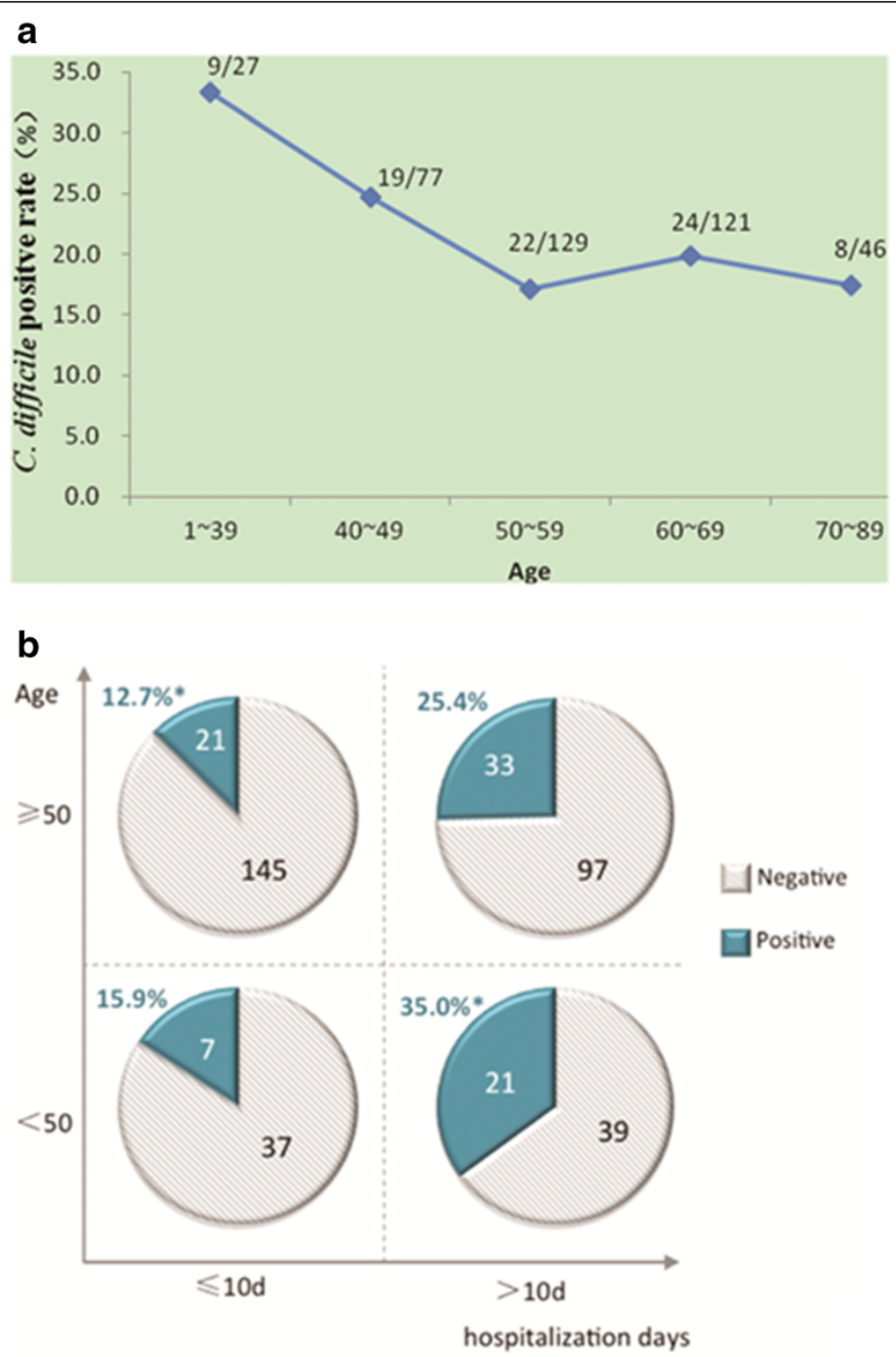

Figure 2 Association of $\boldsymbol{C}$. difficile positive rate with different age and hospitalization days. a. Association between C. difficile positive rate and different age groups. Note: Patients were categorized into five groups of different ages, showing in the figure as positive number/total number of samples in each group. Patients younger than 50 years old had a significantly higher positive rate than the older groups ( $p=0.04)$. b. Subdivision of $C$. difficile positive rate by age and days of hospitalization. Note: Patients were organized into two age groups by the cutoff value of 50 years old. Each age group was then subdivided into two groups based on the number of days in hospital ( $\leq 10$ days or $>10$ days). *: The $C$. difficile positive rate was significantly different between the group that was $<50$ years old and hospitalized $>10$ days compared to the group that was $\geq 50$ years old and hospitalized $\leq 10$ days $(35.0 \%$ vs. $12.7 \%, p=0.0009)$.

the positive rate was as high as $35 \%$ in patients younger than 50 years old who were hospitalized for at least 10 days. In contrast, the positive rate was only $12.7 \%$ in patients older than 50 years old who were hospitalized for less than 10 days. The difference was highly significant $\left(x^{2}=16.5, \mathrm{p}=0.0009\right)$.

\section{PCR ribotyping of the positive strain}

The ribotypes of the $82 \mathrm{C}$. difficile samples were determined. The results yielded 12 ribotype patterns, including ribotype $001(n=22)$, ribotype $017(n=15)$, ribotype 017/1 $(n=16)$, ribotype $014 / 0(n=12)$, ribotype $017 / 2(n=10)$, and one each of ribotypes $666,650,555,445,220,037$, and 087. None of the strains belonged to ribotype 027 .

\section{Immunological indicators and C. difficile-positive rate}

The albumin level, white blood cell count, and concentrations of PGE2, TGF- $\beta$, and IL-10 are important immunological indicators for cancer patients. These indicators were tested for their association with $C$. difficile carriage. 
All of the factors, including white blood cell count, albumin count, PGE2, TGF- $\beta$, and IL-10 concentrations, were not significantly different between the $C$. difficile-positive and negative patients.

\section{Discussion}

In this study, we conducted a comprehensive investigation of $C$. difficile carriage among cancer patients from three hospitals in eastern China. The overall $C$. difficilepositive rate of the cancer patients enrolled in the 7 -month period was $20.5 \%$. This rate is much higher than that (12.6\%) reported in non-cancer patients in a similar study in 2009 in eastern China [14]. The relationship between various clinical and immunological factors and C. difficile carriage was further analyzed. Two factors (age, days of hospitalization) were determined to be significantly associated with the rate of $C$. difficile carriage. First, we found that cancer patients with $C$. difficile carriage appeared to be younger than patients from the U.S. [15]. This difference could be caused by various factors, such as diet, geographical location, intake of food supplements and drugs, and other causes. Studies also have found that features of gut microbiomes are always unique to different locations and lifestyles [16]. The lifestyles of Chinese people, especially those of young Chinese, have changed dramatically over the last 20 years, partially because of the rapid growth of the Chinese economy. The changed lifestyle which was not so common in China in the past, might be a major contributor to the increasing emergence of $C$. difficile infection. Interestingly, the age predisposition was consistent with a previous study of cancer patients in Beijing, China [17]. Meanwhile, a large scale study in Japan also revealed that long hospital stay was associated with $C$. difficile infection [18]. However, these two studies did not provide a cutoff value for age and the hospitalization days. In our study, we found that the cancer patients who were younger than 50 years old and had stayed in hospital for more than 10 days were more prone to $C$. difficile carriage. It is easier to understand that longer stay in hospital exposes a higher risk of infection to patients; it is less clear to us why the younger patients in our study were more prone to $C$. difficile carriage. A similar result was reported recently in northern China [17].

Immune response is important for $C$. difficile carriage. However, among white blood cell count, albumin count, and levels of PGE2, TGF- $\beta$, and IL-10, which are thought to be inhibitors of immunological activity [19], we found no factor was related to the $C$. difficile-positive rate. And ribotyping of $C$. difficile showed that there was no 027 strain, which was the major cause of the deadly C. difficile infection emergence in Canada between 2002 and 2005 [20].

Strikingly, more than $90 \%$ of the C. difficile-positive cancer patients in our study had no symptoms of diarrhea.
The reason might be complicated. A similar study also showed no outbreak of $C$. difficile infection despite C. difficile carriage [21]. Nevertheless, researchers have suggested that asymptomatic carriers can contribute to C. difficile transmission in hospitals [22]. As a result of the near-indefinite viability of the bacteria and the low infective dose, $C$. difficile could be widely transmitted within hospitals in the presence of the ever increasing asymptomatic carriers [23].

\section{Conclusions}

In our current study, no cancer-specific factors were identified to be related to $C$. difficile carriage. However, a younger age and a longer hospitalization stay may represent the characteristics of more aggressive and immunosuppressive oncologic disease. Larger sample size and cancer-specific information mining research is needed in the future in order to clarify the exact role of $C$. difficile carriage in cancer patients.

\section{Abbreviations}

C. difficile: Clostridium difficile; PGE2: Prostaglandin E2; IL-10: interleukin-10; TGF- $\beta$ : Transforming growth factor beta; PPIs: Proton pump inhibitors; CCFA: Cefoxitin-cycloserine fructose agar; ELISA: Enzyme-linked immunosorbent assay.

\section{Competing interests}

The authors declare that they have no competing interests.

\section{Authors' contributions}

FWJ and ZSS designed the study; FWJ, JDZ and LY drafted the manuscript and carried out the molecular genetic studies; ZP, QJ, TBR, CXG, ZYL, ZY, DJ, ZWH, FXR collected the clinical samples; LFL and MXZ performed the statistical analysis. All authors read and approved the final manuscript.

\section{Acknowledgements}

We thank Prof. Ping Fang from Zhejiang University for her help with the statistical analysis. We also thank Mr. Xuexing Wang from Sinyoo Information Technology Company (Shanghai) for his technical support. This study was supported by the Major Scientific Project of Zhejiang Province (2012C13014-2). We would also like to express our gratitude to the Consulting Project of the Chinese Academy of Engineering (2012-XY-12-4), the Natural Science Foundation of China (No. 81471998, No. 81472210), and Zhejiang Natural Science Foundation (LY13B020003) for their support to our study.

\section{Author details}

${ }^{1}$ First Affiliated Hospital, School of Medicine, ZheJiang University, 79 Qinchun Road, Hangzhou 310006, China. '2Department of Microbiology, Zhejiang Provincial Center for Disease Control and Prevention, Hangzhou, China. ${ }^{3}$ Lab of Proteomics \& Molecular Enzymology, School of Life Sciences, Zhejiang Sci-Tech University, Hangzhou 310018, China. ${ }^{4}$ Department of Oncology, Yuyao Hospital, Yuyao, China. ${ }^{5}$ Huzhou Central Hospital, Huzhou, China. ${ }^{6}$ Zhejiang Provincial People's Hospital, Hangzhou, China.

Received: 16 July 2014 Accepted: 25 September 2014 Published: 29 September 2014

\section{References}

1. Ananthakrishnan $A N$ : Clostridium difficile infection: epidemiology, risk factors and management. Nat Rev Gastroenterol Hepatol 2011, 8(1):17-26.

2. Kuijper EJ, van Dissel JT: Spectrum of Clostridium difficile infections outside health care facilities. CMAJ 2008, 179(8):747-748.

3. Garey KW, Dao-Tran TK, Jiang ZD, Price MP, Gentry LO, Dupont HL: A clinical risk index for Clostridium difficile infection in hospitalised 
patients receiving broad-spectrum antibiotics. J Hosp Infect 2008, 70(2):142-147

4. Biagi E, Nylund L, Candela M, Ostan R, Bucci L, Pini E, Nikkila J, Monti D, Satokari R, Franceschi C, Brigidi P, De Vos W: Through ageing, and beyond: gut microbiota and inflammatory status in seniors and centenarians. Plos one 2010, 5(5):e10667.

5. DuPont HL: The search for effective treatment of Clostridium difficile infection. N Engl J Med 2011, 364(5):473-475.

6. Chopra T, Alangaden GJ, Chandrasekar P: Clostridium difficile infection in cancer patients and hematopoietic stem cell transplant recipients. Expert Rev Anti Infect Ther 2010, 8(10):1113-1119.

7. Bartlett JG, Gerding DN: Clinical recognition and diagnosis of Clostridium difficile infection. Clin Infect Dis 2008, 46(Suppl 1):S12-S18.

8. Cohen SH, Gerding DN, Johnson S, Kelly CP, Loo VG, McDonald LC, Pepin J, Wilcox MH, Society for Healthcare Epidemiology of A, Infectious Diseases Society of A: Clinical practice guidelines for Clostridium difficile infection in adults: 2010 update by the society for healthcare epidemiology of America (SHEA) and the infectious diseases society of America (IDSA). Infect Control Hosp Epidemiol 2010, 31(5):431-455.

9. Loo VG, Poirier L, Miller MA, Oughton M, Libman MD, Michaud S, Bourgault AM, Nguyen T, Frenette C, Kelly M, Vibien A, Brassard P, Fenn S, Dewar K, Hudson TJ, Horn R, Rene P, Monczak Y, Dascal A: A predominantly clonal multi-institutional outbreak of Clostridium difficile-associated diarrhea with high morbidity and mortality. N Engl J Med 2005, 353(23):2442-2449.

10. McDonald LC, Killgore GE, Thompson A, Owens RC Jr, Kazakova SV, Sambol SP, Johnson S, Gerding DN: An epidemic, toxin gene-variant strain of Clostridium difficile. N Engl J Med 2005, 353(23):2433-2441.

11. Lemee L, Dhalluin A, Testelin S, Mattrat MA, Maillard K, Lemeland JF, Pons JL: Multiplex PCR targeting tpi (triose phosphate isomerase), tcdA (Toxin A), and tcdB (Toxin B) genes for toxigenic culture of Clostridium difficile. J Clin Microbiol 2004, 42(12):5710-5714.

12. Indra A, Blaschitz M, Kernbichler S, Reischl U, Wewalka G, Allerberger F: Mechanisms behind variation in the Clostridium difficile 16S-23S rRNA intergenic spacer region. J Med Microbio/ 2010, 59(Pt 11):1317-1323.

13. Indra A, Huhulescu $S$, Schneeweis M, Hasenberger $P$, Kernbichler $S$, Fiedler A, Wewalka G, Allerberger F, Kuijper EJ: Characterization of Clostridium difficile isolates using capillary gel electrophoresis-based PCR ribotyping. J Med Microbiol 2008, 57(Pt 11):1377-1382.

14. Huang $H$, Wu S, Wang M, Zhang Y, Fang H, Palmgren AC, Weintraub A, Nord CE: Clostridium difficile infections in a Shanghai hospital: antimicrobial resistance, toxin profiles and ribotypes. Int J Antimicrob Agents 2009, 33(4):339-342.

15. Boone JH, Goodykoontz M, Rhodes SJ, Price K, Smith J, Gearhart KN, Carman RJ, Kerkering TM, Wilkins TD, Lyerly DM: Clostridium difficile prevalence rates in a large healthcare system stratified according to patient population, age, gender, and specimen consistency. Eur J Clin Microbiol Infect Dis 2012, 31(7):1551-1559.

16. Yatsunenko T, Rey FE, Manary MJ, Trehan I, Dominguez-Bello MG, Contreras M, Magris M, Hidalgo G, Baldassano RN, Anokhin AP, Heath AC, Warner B, Reeder J, Kuczynski J, Caporaso JG, Lozupone CA, Lauber C, Clemente JC, Knights D, Knight R, Gordon Jl: Human gut microbiome viewed across age and geography. Nature 2012, 486(7402):222-227.

17. Han XH, Du CX, Zhang CL, Zheng CL, Wang L, Li D, Feng Y, DuPont HL, Jiang ZD, Shi YK: Clostridium difficile infection in hospitalized cancer patients in Beijing, China is facilitated by receipt of cancer chemotherapy. Anaerobe 2013, 24:82-84.

18. Yasunaga $H$, Horiguchi $H$, Hashimoto $H$, Matsuda S, Fushimi $K$ : The burden of Clostridium difficile-associated disease following digestive tract surgery in Japan. J Hosp Infect 2012, 82(3):175-180.

19. Lee $\mathrm{CH}$, Chen RF, Liu JW, Yeh WT, Chang JC, Liu PM, Eng HL, Lin MC, Yang KD: Altered p38 mitogen-activated protein kinase expression in different leukocytes with increment of immunosuppressive mediators in patients with severe acute respiratory syndrome. J Immunol 2004, 172(12):7841-7847

20. Pepin J, Valiquette L, Cossette B: Mortality attributable to nosocomial Clostridium difficile-associated disease during an epidemic caused by a hypervirulent strain in Quebec. CMAJ 2005, 173(9):1037-1042.

21. Chen YB, Gu SL, Wei ZQ, Shen P, Kong HS, Yang Q, Li L: Molecular epidemiology of Clostridium difficile in a tertiary hospital of China. J Med Microbiol 2014, 63(Pt 4):562-569.
22. Guerrero DM, Becker JC, Eckstein EC, Kundrapu S, Deshpande A, Sethi AK, Donskey CJ: Asymptomatic carriage of toxigenic Clostridium difficile by hospitalized patients. J Hosp Infect 2013, 85(2):155-158.

23. Curry SR, Muto CA, Schlackman JL, Pasculle AW, Shutt KA, Marsh JW Harrison LH: Use of multilocus variable number of tandem repeats analysis genotyping to determine the role of asymptomatic carriers in Clostridium difficile transmission. Clin Infect Dis 2013, 57(8):1094-1102.

doi:10.1186/1471-2334-14-523

Cite this article as: Fang et al:: Clostridium difficile carriage in hospitalized cancer patients: a prospective investigation in eastern China. BMC Infectious Diseases 2014 14:523.

\section{Submit your next manuscript to BioMed Central and take full advantage of:}

- Convenient online submission

- Thorough peer review

- No space constraints or color figure charges

- Immediate publication on acceptance

- Inclusion in PubMed, CAS, Scopus and Google Scholar

- Research which is freely available for redistribution

Submit your manuscript at www.biomedcentral.com/submit 